Wilfrid Laurier University

Scholars Commons @ Laurier

Physics and Computer Science Faculty

Publications

Physics and Computer Science

1992

\title{
Well-Behaved Dynamics in a Parametrically Damped Pendulum
}

\author{
Binrou Wu \\ University of Waterloo \\ James A. Blackburn \\ Wilfrid Laurier University, jabjabjab@cogeco.ca
}

Follow this and additional works at: https://scholars.wlu.ca/phys_faculty

\section{Recommended Citation}

Wu, Binrou and Blackburn, James A., "Well-Behaved Dynamics in a Parametrically Damped Pendulum" (1992). Physics and Computer Science Faculty Publications. 58.

https://scholars.wlu.ca/phys_faculty/58

This Article is brought to you for free and open access by the Physics and Computer Science at Scholars Commons @ Laurier. It has been accepted for inclusion in Physics and Computer Science Faculty Publications by an authorized administrator of Scholars Commons @ Laurier. For more information, please contact scholarscommons@wlu.ca. 


\title{
Well-behaved dynamics in a parametrically damped pendulum
}

\author{
Binruo Wu \\ Department of Physics, University of Waterloo, Waterloo, Ontario, Canada N2L $3 G 1$
}

James A. Blackburn

Department of Physics and Computing, Wilfrid Laurier University, Waterloo, Ontario, Canada N2L $3 C 5$

(Received 18 November 1991)

\begin{abstract}
Well-behaved dynamical properties have been found in a parametrically damped pendulum. For various dampings, all minimum forcing amplitudes $E$ for chaos to occur are at the forcing frequency $\Omega=1.66$, and all minimum $E$ for a stationary solution to be unstable are identical $(E=2)$ and at $\Omega=2$ in the $\Omega-E$ state space. Between these two frequencies, the variation to chaos along stability boundaries (where the stationary solution becomes unstable) is solely $\Omega$ dependent and insensitive to dampings. These two frequencies separate parameter regions with distinct dynamical behaviors. For $\Omega<1.66$, the route to chaos is due to an inverse boundary crisis while for $\Omega>1.66$, it is associated with period doubling. For $\Omega<2$ the transition from stationary solution to periodic solution is a jump, while for $\Omega>2$ it is a Hopf bifurcation.

PACS number(s): 05.45. $+\mathrm{b}, 03.20 .+\mathrm{i}$
\end{abstract}

\section{INTRODUCTION}

Beginning with a damped but unforced pendulum, an external excitation may be introduced in three distinctly different ways. The first is the well-known forced and damped pendulum,

$$
\ddot{x}+\gamma \dot{x}+\sin (x)=F \sin (\Omega t),
$$

the second is a damped pendulum with its pivot subjected to vertical oscillations,

$$
\ddot{x}+\gamma \dot{x}[1+E \sin (\Omega t)] \sin (x)=0,
$$

and the third is a parametrically damped pendulum,

$$
\ddot{x}+\gamma[1+E \sin (\Omega t)] \dot{x}+\sin (x)=0,
$$

where $x$ and $\dot{x}$ represent the angular position and velocity, respectively, $\gamma$ is the damping coefficient, and $F$ (or $E$ ) and $\Omega$ are the external forcing amplitude and frequency, respectively. All variables and parameters are expressed in dimensionless forms.

In contrast to Eq. (1), Eqs. (2) and (3) admit a stationary solution $x=\dot{x}=0$. Stability boundaries defined by the onset of instability of this zero solution will arise in these latter two cases.

In their investigations of Eq. (3), Smith and Blackburn [1] numerically obtained a $V$-shape stability boundary for $\gamma=\frac{1}{18.33}$, which was roughly symmetric about the vertical line $\Omega=2$ in the $\Omega-E$ state space. Another $V$-shape chaotic boundary was situated inside the stability boundary, with their left sides well aligned. It is not clear if these features reflect well-behaved dynamical properties, i.e., they occur not just for specific parameters. Some commonly concerned questions, such as what the routes to chaos are and how the stationary solutions become unstable, also need to be answered. Therefore a further systematic analysis and, especially, an analytical formula for stability boundaries are desirable.

However, an analytical discussion may not be possible for this highly nonlinear system without approximation. As a classical example, Eq. (1) can be treated by perturbation and averaging only after the Taylor series of $\sin (x)$ has been truncated, say, the Duffing equation $[2,3]$. The Mathieu equation, the linearization of Eq. (2), is analytically solvable for its stability boundaries [4]. It is not clear if these boundaries are also for Eq. (2) itself. In this paper, a main conclusion has been reached that the stability boundaries of Eq. (3) can be obtained by analytically solving its linearized equation. This conclusion has, in return, shown that the stability boundaries of the Mathieu equation indeed serve Eq. (2) as well. It is valuable to reach such a conclusion for these highly nonlinear systems.

Besides other fine dynamical properties, it will be demonstrated in this paper that the variation of periodic solutions along left sides of stability boundaries from $\Omega=2$ to 1.66 is insensitive to dampings. That is, the solutions are of identical periods and shapes in phase space at a fixed forcing frequency in spite of different dampings. It is this regularity that results in the occurrence of all the lowest transitions to chaos at $\Omega=1.66$.

It would seem natural to consider the application of Melnikov's method to predict the chaotic boundary for Eqs. (1)-(3). Unfortunately, unlike its partially successful application to chaos occurring in Eq. (1) when damping and forcing are small [5-7], this method is not suitable for Eq. (3) when chaos occurs at large forcing values.

\section{STABILITY BOUNDARIES FOR STATIONARY SOLUTION}

To test the stability of the stationary solution of Eq. (3), an arbitrary initial perturbation $(\delta x, \delta \dot{x})$ to the origin of the phase space $(x, \dot{x})$ is assumed. Because $\delta x=x$ and 
$\delta \dot{x}=\dot{x}$ with respect to the origin, the validity of $\sin (x) \approx x$ and the smallness of the term $\gamma(1+E \sin \Omega t) \dot{x}$ in Eq. (3) are guaranteed. This simple but very important point has distinguished Eq. (3) from, for example, Eq. (1), where any perturbations $\delta x$ and $\delta \dot{x}$ are with respect to certain values of $x$ and $\dot{x}$ and thus extra care is needed in considering the validity of linearization. It should be emphasized that this perturbation is appropriate only for stability boundaries, instead of periodic solutions, for Eq. (3).

The method of Lindstedt-Poincaré perturbation has been chosen. Let $\gamma=\epsilon \beta$ and $\Omega t=\tau$, and replace $\sin (x)$ with $x$. Equation (3) then becomes

$$
\Omega^{2} \ddot{x}+\epsilon \beta \Omega(1+E \sin \tau) \dot{x}+x=0,
$$

where the time derivative is with respect to $\tau$.

Set

$$
\begin{aligned}
& \Omega=\Omega_{0}+\epsilon \Omega_{1}+\epsilon^{2} \Omega_{2}+O\left(\epsilon^{3}\right), \\
& x=x_{0}+\epsilon x_{1}+\epsilon^{2} x_{2}+O\left(\epsilon^{3}\right) .
\end{aligned}
$$

By substituting the above expressions for $x$ and $\Omega$ into Eq. (4), we have

$$
\begin{aligned}
\Omega_{0}^{2} \ddot{x}_{0}+x_{0}= & 0\left(\epsilon^{0}\right), \\
\Omega_{0}^{2} \ddot{x}_{1}+x_{1}= & -2 \Omega_{0} \Omega_{1} \ddot{x}_{0}+\beta(1+E \sin \tau) \Omega_{0} \dot{x}_{0} \quad\left(\epsilon^{1}\right), \\
\Omega_{0}^{2} \ddot{x}_{2}+x_{2}= & -\left(2 \Omega_{0} \Omega_{2}+\Omega_{1}^{2}\right) \ddot{x}_{0}-2 \Omega_{0} \Omega_{1} \ddot{x}_{1} \\
& -\beta(1+E \sin \tau)\left(\Omega_{1} \dot{x}_{0}+\Omega_{0} \dot{x}_{1}\right) \quad\left(\epsilon^{2}\right) .
\end{aligned}
$$

From Eq. (5), the zeroth solution to Eq. (4) is

$$
x_{0}=a \sin \left(\frac{1}{\Omega_{0}} \tau\right)+b \cos \left(\frac{1}{\Omega_{0}} \tau\right) \text {. }
$$

Substituting Eq. (8) into Eq. (6), we obtain

$$
\begin{aligned}
\Omega_{0}^{2} \ddot{x}_{1}+x_{1}= & \left(2 \frac{\Omega_{1}}{\Omega_{0}} a-\beta b\right) \sin \left(\frac{1}{\Omega_{0}} \tau\right)+\left(2 \frac{\Omega_{1}}{\Omega_{0}} b+\beta a\right) \cos \left[\frac{1}{\Omega_{0}} \tau\right] \\
& +\frac{1}{2} \beta E a\left[\sin \left[1+\frac{1}{\Omega_{0}}\right] \tau+\sin \left(1-\frac{1}{\Omega_{0}}\right) \tau\right]+\frac{1}{2} \beta E b\left[\cos \left(1+\frac{1}{\Omega_{0}}\right) \tau-\cos \left(1-\frac{1}{\Omega_{0}}\right) \tau\right]
\end{aligned}
$$

To eliminate the secular terms in Eq. (9) when $\Omega_{0} \neq 2$, we must have $a=b=0$, which means only the periodic solutions with period 2 exist. When $\Omega_{0}=2$, for nonzero $a$ and $b$, we must have

$$
\Omega_{1}= \pm \beta\left(\frac{1}{4} E^{2}-1\right)^{1 / 2},
$$

where $E \geq 2$. Equation (10) is the formula of the stability boundaries to first order when $\Omega_{1}$ is replaced with $(\Omega-2)$. Equation (9) now becomes

$$
\Omega_{0}^{2} \ddot{x}_{1}+x_{1}=\frac{1}{2} \beta E a \sin \left(\frac{3}{2} \tau\right)+\frac{1}{2} \beta E b \cos \left(\frac{3}{2} \tau\right) .
$$

Solving Eq. (11) for $x_{1}$ :

$$
x_{1}=-\frac{1}{16} \beta E\left[a \sin \left(\frac{3}{2} \tau\right)+b \cos \left(\frac{3}{2} \tau\right)\right] .
$$

Substituting Eq. (12) into Eq. (7) we will obtain an equation for $x_{2}$. To eliminate the secular terms in that equation, the following conditions must be met:

$$
\begin{aligned}
& {\left[\Omega_{2}+\frac{1}{4} \Omega_{1}^{2}+\frac{1}{\beta} E \Omega_{1}+\frac{3}{16} \beta^{2} E^{2}\right] a-\frac{1}{2} \beta \Omega_{1} b=0,} \\
& \frac{1}{2} \beta \Omega_{1} a-\left[\Omega_{2}+\frac{1}{4} \Omega_{1}^{2}-\frac{1}{\beta} E \Omega_{1}+\frac{3}{16} \beta^{2} E^{2}\right] b=0 .
\end{aligned}
$$

For nontrivial solutions of $a$ and $b$, the Wronskian of Eq. (13) must be zero, thus

$$
\left(\Omega_{2}+\frac{1}{4} \Omega_{1}^{2}+\frac{3}{16} \beta^{2} E^{2}\right)^{2}-\frac{1}{4} \beta^{2} \Omega_{1}^{2}\left(\frac{1}{4} E^{2}-1\right)=0 .
$$

Substitute Eq. (10) into Eq. (14) to get

$$
\begin{aligned}
& \Omega_{2}=-\frac{9}{16} \beta^{2} E^{2}+\frac{3}{4} \beta^{2}, \\
& \Omega_{2}=-\frac{1}{16} \beta^{2} E^{2}-\frac{1}{4} \beta^{2} .
\end{aligned}
$$

We choose Eq. (15b) because the result obtained by Eq. (15a) is too large for a second-order modification to $\Omega$. In terms of Eqs. (10) and (15b), we have

$$
\begin{aligned}
\Omega & =\Omega_{0}+\epsilon \Omega_{1}+\epsilon^{2} \Omega_{2}+O\left(\epsilon^{3}\right) \\
& =2 \pm \epsilon \beta\left(\frac{1}{4} E^{2}-1\right)^{1 / 2}+\epsilon^{2} \beta^{2}\left(-\frac{1}{16} E^{2}-\frac{1}{4}\right)+O\left(\epsilon^{3}\right) \\
& =2 \pm \gamma\left(\frac{1}{4} E^{2}-1\right)^{1 / 2}+\gamma^{2}\left(-\frac{1}{16} E^{2}-\frac{1}{4}\right)+O\left(\epsilon^{3}\right) .
\end{aligned}
$$

Hence we have obtained the final formulas for the stability boundary:

$\Omega=2+\gamma\left(\frac{1}{4} E^{2}-1\right)^{1 / 2}-\gamma^{2}\left(\frac{1}{16} E^{2}+\frac{1}{4}\right)$ for $\Omega>2$,

$\Omega=2-\gamma\left(\frac{1}{4} E^{2}-1\right)^{1 / 2}-\gamma^{2}\left(\frac{1}{16} E^{2}+\frac{1}{4}\right)$ for $\Omega<2$.

When $\Omega=2$, by equating Eqs. (16a) and (16b), we have $E=2$. Note this is the common minimum transition value for all $\gamma$ 's.

For applying this formula at given $\Omega$ and $\gamma$, only simple iterations are needed to find the corresponding $E$. Figures 1 (a) and 1(b) show comparisons between the stability boundaries obtained by numerical calculation, first-order perturbation, and second-order perturbation, for $\gamma=\frac{1}{18.33}$ and $\frac{1}{5}$, corresponding to light and intermediate dampings, respectively. It is evident that Eqs. (16a) and (16b) are quite accurate. 

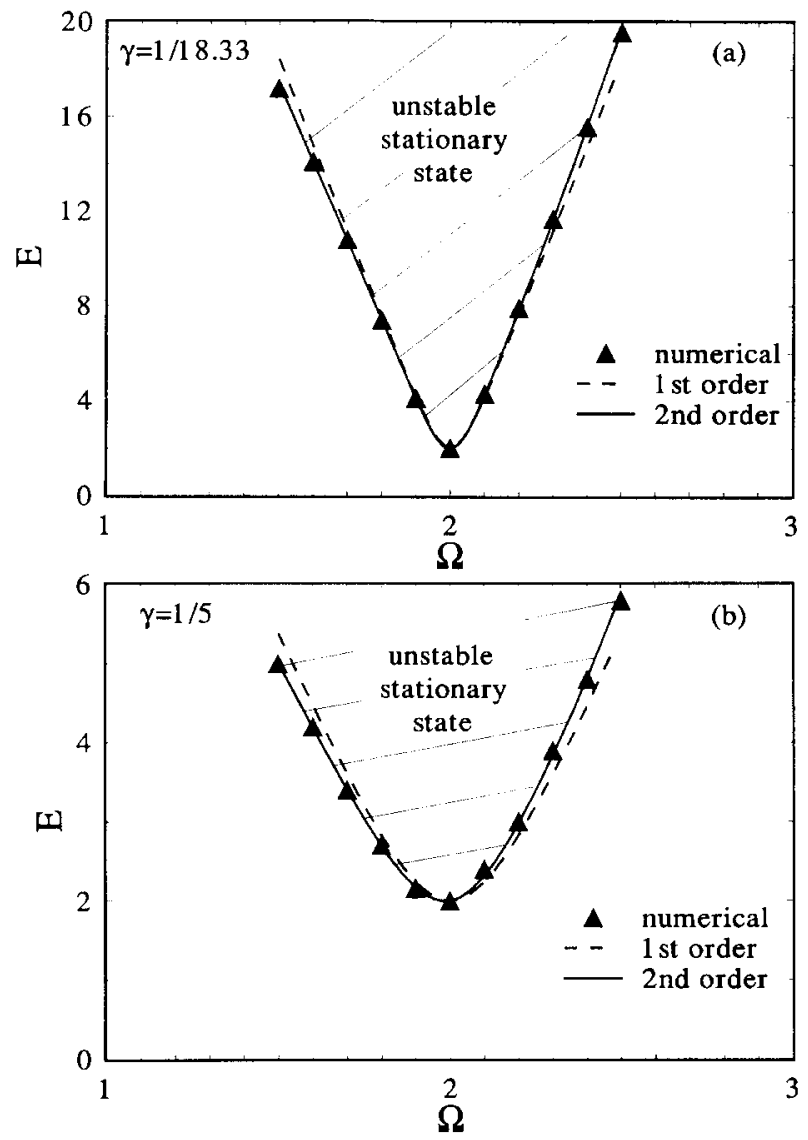

FIG. 1. Stability boundaries in the $\Omega-E$ state space: (a) for $\gamma=\frac{1}{18.33}$ and (b) for $\gamma=\frac{1}{5}$, respectively. The results by the numerical simulations, the first-order perturbation, and the second-order perturbation are shown and compared.

\section{CHAOTIC BOUNDARIES DETERMINED BY LYAPUNOV EXPONENTS}

For our three-dimensional, non-Hamiltonian system Eq. (3), the Lyapunov spectra would be $(+, 0,-)$ when it is chaotic. To indicate the onset of chaos, we only need to calculate the largest Lyapunov exponent. Based on the method developed by Shimada and Nagashima [8], as well as by Wolf et al. [9], an interactive computer program was written for this purpose.

As indicated in Ref. [1], transient chaos is likely to occur just below the upper-left-side stability boundary. An estimate of the positive Lyapunov exponent when transient chaos is not over will indicate a lower chaotic boundary in that area. Hence an initial interval of 2000 forcing periods is computed before we begin the evaluation of another 2000 forcing periods. To determine a possible chaotic trajectory accurately over such a long time, a higher-order Runge-Kutta-Verner algorithm, the RKV-7 [10], was selected. Grebogi et al. [11] reported that, for a driven Hamiltonian pendulum, a noisy trajectory is shadowed by a true trajectory with slightly

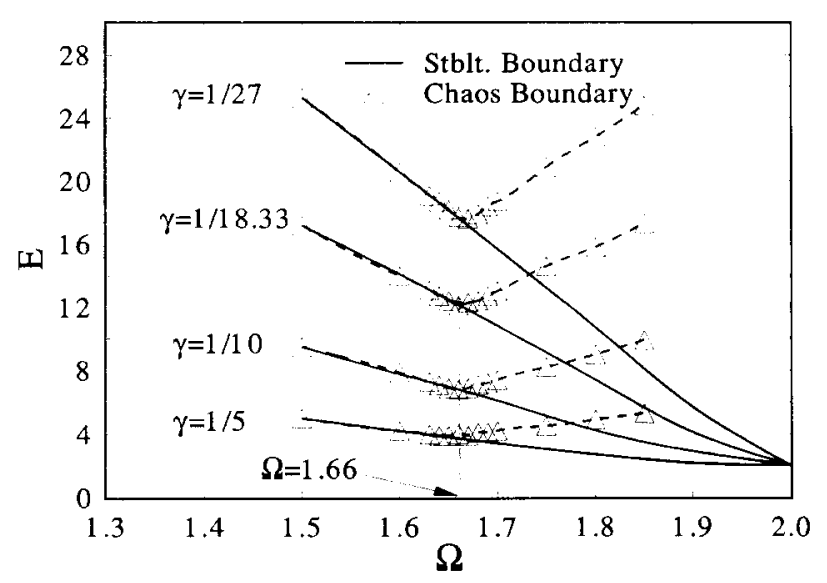

FIG. 2. The left-side stability boundaries and the chaotic boundaries in the $\Omega-E$ state space for $\gamma=\frac{1}{5}, \frac{1}{10}, \frac{1}{18.33}$, and $\frac{1}{27}$, respectively. The chaotic boundaries determined by positive Lyapunov exponents are marked by the triangles. The transition boundaries marked by the solid lines are obtained from the second-order perturbation.

different initial conditions within the distance of $10^{-8}$ for the computation time up to 5000 forcing cycles, by using a seventh-order integration method. Compared with their results, we believe that the chaotic boundaries calculated by our program are reliable.

These chaotic boundaries are indicated by the empty triangles in Fig. 2 for four different dampings. This figure reveals that the minimum $E$ 's of these boundaries occur at a frequency $\Omega=1.66$, while the stability boundaries all have a minimum at $\Omega=2$ and $E=2$. This figure also shows that the chaotic boundaries coincide with the transition boundaries for $\Omega \leq 1.66$. It will be demonstrated that the transition mode for $\Omega<2$ is a jump from the stationary solution to periodic solutions. It is possible that this jump may be a leap into chaos rather than to a periodic solution. This route to chaos can be categorized as an inverse boundary crisis [12]. Such a crisis has been detected in Eq. (1) by Kautz and MacFarlane [13]. A similar coincidence between the jump boundary and the hopping boundary to chaos for Eq. (1) was earlier reported [14]. We may thus conclude that chaotic boundaries due to crises in systems of the pendulum type could be predicted by locating their jump boundaries.

\section{TWO BIFURCATION MODES AT STABILITY BOUNDARIES}

Based on a symmetry analysis, as applied to Eq. (1) by Kerr et al. [15], the periodic solution of Eq. (3) will be composed by a series of harmonics (we suppose no subharmonic bifurcation): $\frac{1}{2} \Omega, \frac{3}{2} \Omega, \frac{5}{2} \Omega, \ldots$ The constant component and all even harmonics will be missing. Then a stable periodic solution at the transition boundaries may be approximately expressed as

$x=a \sin \left(\frac{1}{2} \Omega t\right)+b \cos \left(\frac{1}{2} \Omega t\right)=r \sin \left[\frac{1}{2}(\Omega t+\phi)\right]$, 
where $r=\left(a^{2}+b^{2}\right)^{1 / 2}$, and $\phi=\arctan (b / a)$. Substituting Eq. (17) into Eq. (3) and using the Fourier-Bessel identity

$$
\begin{aligned}
\sin \left\{r \sin \left[\frac{1}{2}(\Omega t+\phi)\right]\right\}= & 2 J_{1}(r) \sin \left[\frac{1}{2}(\Omega t+\phi)\right] \\
& +2 J_{3}(r) \sin \left[\frac{3}{2}(\Omega t+\phi)\right] \\
& +\cdots,
\end{aligned}
$$

then we obtain the following amplitude equation from harmonic balance [3]:

$$
E=2\left[4 \gamma^{-2} \Omega^{-2}\left[\frac{2 J_{1}(r)}{r}-\frac{\Omega^{2}}{4}\right]^{2}+1\right]^{1 / 2} .
$$

Equation (18) reveals two distinct transition modes. When $\Omega>2, \Omega^{2} / 4>1$, so $E(r=0)$ is its minimum [the maximum value of $2 J_{1}(r) / r$ is 1 when $\left.r=0\right]$. $E(r>0)$ will monotonically increase while $r$ increases. It is quite different when $\Omega<2$. Now $\Omega^{2} / 4<1$ and $E(r=0)$ is a local maximum. $E$ will first decrease to 2 , then rise again
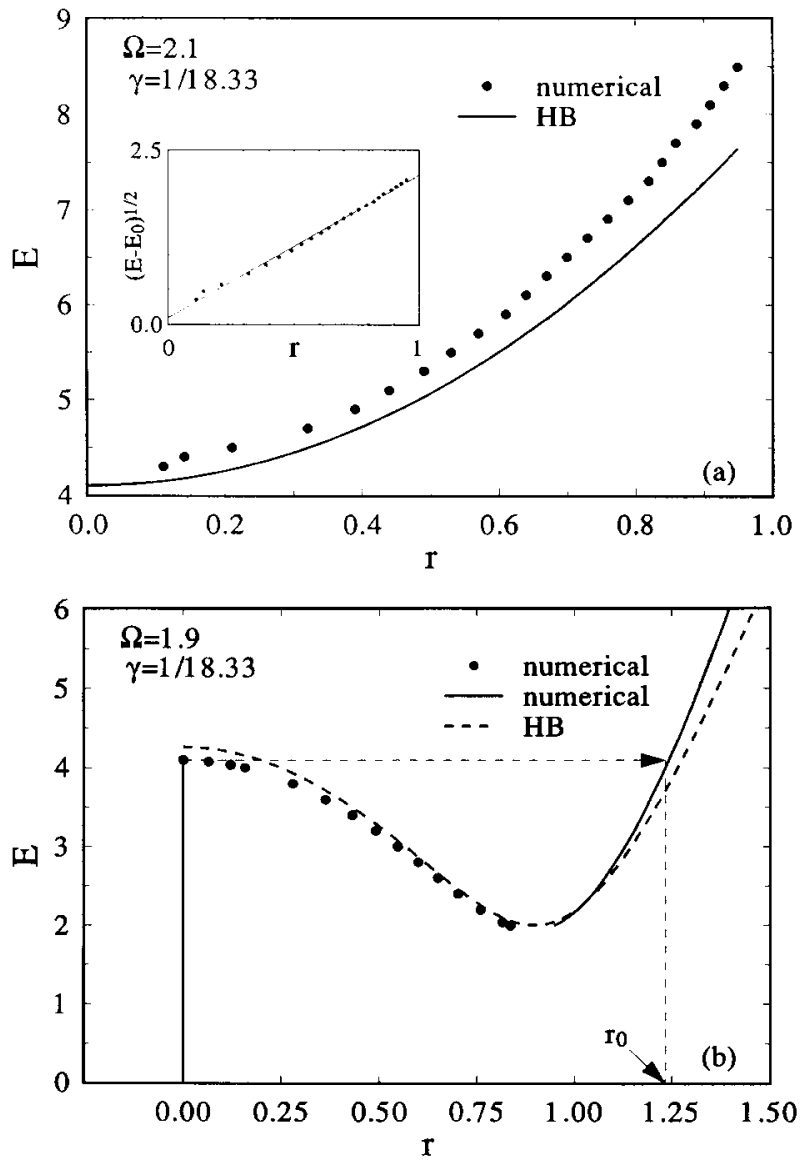

FIG. 3. Two bifurcation modes at stability boundaries. (a) The Hopf bifurcation for $\Omega>2$. The results obtained by harmonic balance (HB) and numerical simulations are shown and compared. The inset is a fit to the numerical data in order to show that $r \sim\left(E-E_{0}\right)^{1 / 2}$. (b) The jump bifurcation for $\Omega<2$. The unstable periodic solutions marked by the solid circles are obtained by the Newton-Raphson method. as $r$ continues to increase. This gradual increase of $r$ is not physically possible because there will be a jump from $r=0$ to $r_{0}$ under the condition $E(r=0)=E\left(r=r_{0}\right)$. These two cases (one for $\Omega=2.1$ and the other for $\Omega=1.9$ ) are depicted in Figs. 3(a) and 3(b), respectively, where the numerical results are also shown for comparison. The inset of Fig. 3(b) is a regression curve of $\left(E-E_{0}\right)^{1 / 2} \sim r$ where $E_{0}$ is obtained from Eq. (16a),
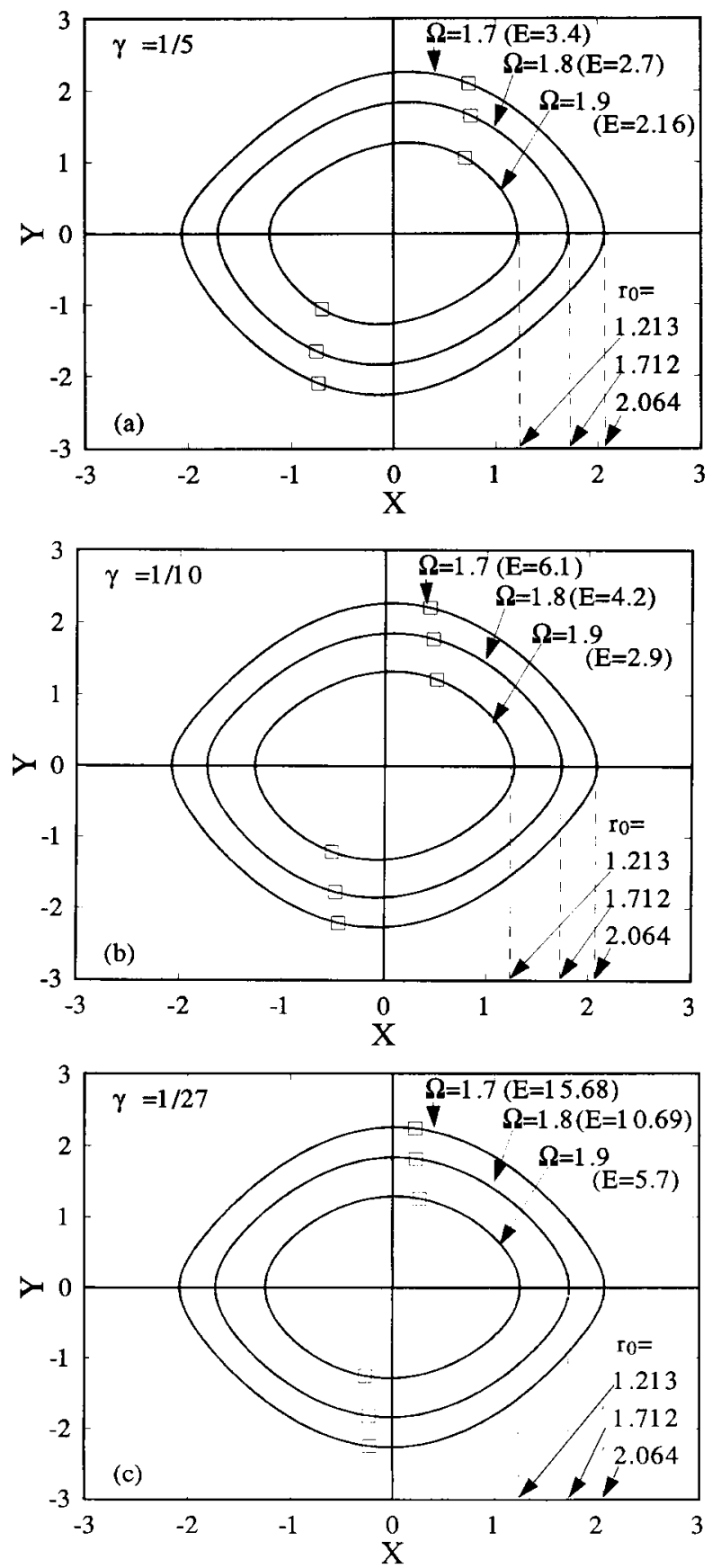

FIG. 4. Phase plots after the jump at $\Omega=1.9,1.8$, and 1.7 for $\gamma=\frac{1}{5}$ (a), $\frac{1}{10}$ (b), and $\frac{1}{27}$ (c), respectively. These pictures clearly show that the amplitude of the solution for $1.7<\Omega<2$ is only $\Omega$ dependent. 


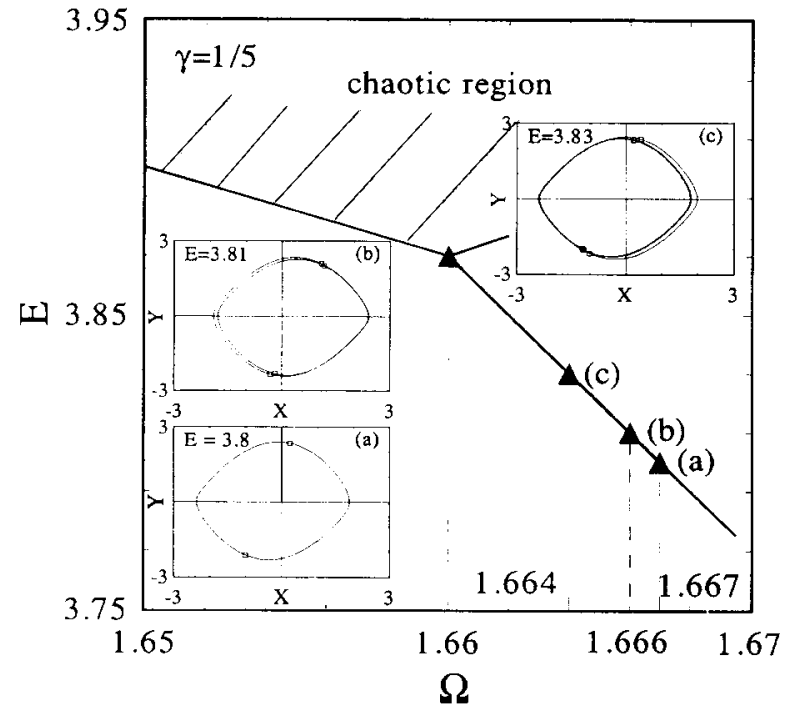

FIG. 5. Numerical results for the transition to chaos along the stability boundary for $1.66<\Omega<1.7$ in the $\Omega-E$ state space. Insets: (a) The jump to a symmetry-breaking period 2 at $\Omega=1.667$; (b) the jump to a period-4 solution at $\Omega=1.666$; and (c) the jump to a phase-locked chaos at $\Omega=1.664$.

which shows that it is indeed a Hopf bifurcation at the transition boundaries for $\Omega>2$.

Another important conclusion which may be drawn is that the jump amplitude $r_{0}$ is only $\Omega$ dependent for $\Omega<2$. It is not difficult to see from Eq. (18) that

$$
\frac{J_{1}\left(r_{0}\right)}{r_{0}}=\frac{\Omega^{2}}{4}-\frac{1}{2}
$$

According to Eq. (19), for $\Omega=1.9,1.8$, and $1.7, r_{0}=1.13$, 1.88 , and 2.37 , respectively. The numerical values in Figs. 4(a), 4(b), and $4(\mathrm{c})$ are for $\Omega=1.9,1.8$, and 1.7, $r_{0}=1.53,1.712$, and 2.064 , respectively. The $r_{0}$ 's show little difference when $\Omega$ is fixed but damping was changed from $\gamma=\frac{1}{5}$ to $\frac{1}{27}$.

Equation (19) is no longer valid for $\Omega<1.7$ where the periodic solutions lose their symmetry in phase space. But the conclusion that the variation of solutions is only $\Omega$ dependent follows from a slightly different argument. For $1.66<\Omega<1.7$, all transitions which take place at a fixed frequency but corresponding to different dampings will be jumps to the states with identical dynamical properties. For example, it is a jump to period 2 with broken symmetry at $\Omega=1.667$, a jump to period 4 at $\Omega=1.666$, and a jump to phase-locked chaos at $\Omega=1.664$, as shown by Figs. 5(a), 5(b) and 5(c). We believe that it is this dependence only on frequency that results in the occurrence of all lowest chaos at $\Omega=1.66$ for various dampings.

\section{TWO ROUTES TO CHAOS}

Equations (1) - (3) may have similar routes to chaos because their unperturbed equations of motion are the same. One route is due to the intersections of the stable and unstable manifolds of the perturbed homoclinic orbit associated with the unforced and undamped pendulum, which, we believe, follows the period-doubling scenario to chaos. The numerical simulations in Refs. [13] and [14] showed that a crisis (or hopping) also could lead to chaos for Eq. (1). With the coincidence between its chaotic boundary and stability boundary for $\Omega \leq 1.66$, Eq. (3) may have one route to chaos by crises for $\Omega \leq 1.66$ and the other period doubling for $\Omega>1.66$.

To confirm this speculation, a Poincaré map has first been made at $\Omega=1.68$, as shown in Fig. 6(a). Starting at $E=3.6$ with the initial conditions $x=0.01$ and $\dot{x}=0$, and taking the transient time as 100 forcing periods after every increment of $\Delta E=0.01$, we sample another 100 forcing periods by picking up the first $x$ value $[x(1)$ in the figure] of every forcing cycle. Figure 6(a) shows that the zero solution becomes unstable at $E=3.61$ and jumps to a symmetric period 2 . Then it undergoes symmetry breaking at $E=3.69$. Period doubling begins at $E=3.92$
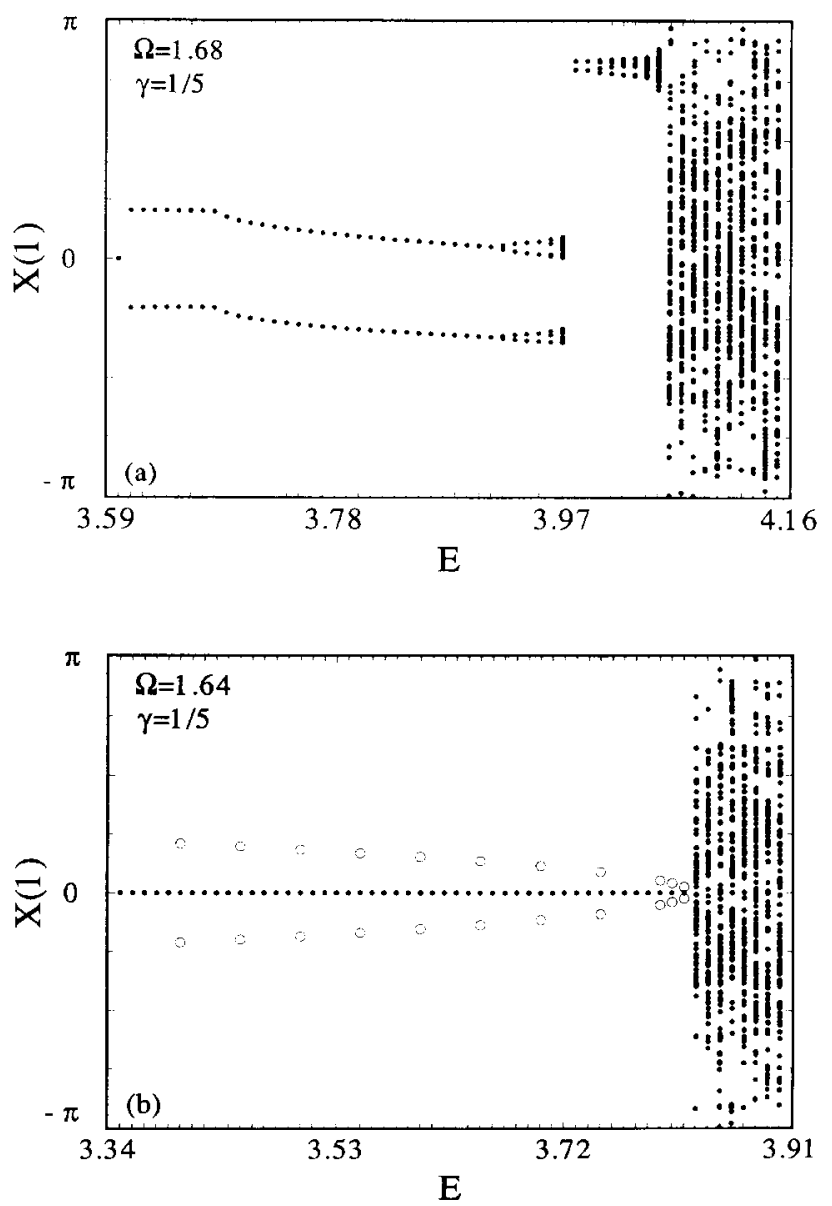

FIG. 6. Two routes to chaos. (a) A route to chaos through period doubling at $\Omega=1.68$, anticipated by a symmetry breaking at $E=3.69$. (b) A route to chaos by the inverse boundary crisis at $\Omega=1.66$. The unstable period 2 collides with the stable stationary solution at $E=3.82$, just before full chaos occurs. 
with the pattern of period $4,8, \ldots$ After the phaselocked chaos appears at $E=3.97$, the system switches to another rotating period 2. The following is also a period doubling which eventually reaches full chaos at $E=4.06$.

A Poincaré map for $\Omega=1.64$ was generated in a slightly different fashion. The initial conditions $x=0.01$ and $\dot{x}=0$ are set for every new $E$ after $\Delta E$ is added. In addition, the transient time has been taken as long as 2000 forcing periods when $E$ approaches the transition boundary $(E \approx 3.8)$. Furthermore, the Newton-Raphson method [16] was used to look for possible unstable solutions in order to find any sign of the collision between stable and unstable solutions. Figure 6(b) shows the result we expected. There is indeed an unstable period-2 solution [it should appear at $E=2$, as Fig. 3(b) shows]. The amplitude of this unstable period 2 continues to decrease until it collides with the stable stationary solution at $E=3.82$. At $E=3.83$, the Poincare data expand to cover the interval $-\pi<x<\pi$, which means that full chaos occurs in a sudden manner. The Lyapunov exponent calculated at $E=3.9$ is 0.176 (the resolution for calculating the Lyapunov exponent is taken to be $\Delta E=0.1$ ).

\section{CONCLUSION}

The well-behaved dynamical properties of the parametrically damped pendulum appear mainly in three aspects. First, the minimum forcing amplitudes $E$ needed to drive this system into chaos are all at the forcing frequency $\Omega=1.66$ in the $\Omega-E$ state space. Secondly, the minimum forcing amplitudes to destabilize the stationary solution are all equal to the same value of $E=2$ and at the forcing frequency $\Omega=2$. Thirdly, at a fixed forcing frequency between $\Omega=2$ and 1.66 , periodic solutions at the stability boundaries corresponding to different dampings are identical in their periods and almost identical in their orbital shapes in phase space. All these properties may be summarized as an insensitivity to damping and it is this behavior that makes this system unique.

\section{ACKNOWLEDGMENTS}

Funding for this research was provided by the Natural Sciences and Engineering Research Council of Canada. The authors gratefully acknowledge enlightening discussions with Dr. H. J. T. Smith.
[1] H. J. T. Smith and James A. Blackburn, Phys. Rev. A 40, 4708 (1989).

[2] X. Nayfeh, Introduction to Perturbation Techniques (Wiley, New York, 1981).

[3] D. W. Jordan and P. Smith Nonlinear Ordinary Differential Equations (Oxford University Press, New York, 1988).

[4] V. I. Arnold, Ordinary Differential Equations (MIT, Cambridge, MA, 1981).

[5] M. Bartuccelli et al., Phys. Rev. B 33, 4686 (1986).

[6] F. M. A. Salam and S. S. Sanstry, IEEE Trans. Circuits Sys. 32, 784 (1985).

[7] G. Cicogna and L. Fronzoni, Phys. Rev. A 42, 1901
(1990).

[8] I. Shimada and T. Nagashima, Prog. Theor. Phys. 61, 1605 (1979).

[9] A. Wolf et al., Physica D 16, 285 (1985).

[10] J. H. Verner, SIAM J. Numerical Anal. 15, 772 (1978).

[11] C. Grebogi et al., Phys. Rev. Lett. 65, 1527 (1990).

[12] C. Grebogi and E. Ott, Physica D 7, 181 (1983).

[13] R. L. Kautz and J. C. MacFarlane, Phys. Rev. A 33, 498 (1986).

[14] Yao Huang Kao, Jeun Chyuan Huang, and Yih Shun Gou, J. Low Temp Phys. 63, 287 (1986).

[15] W. C. Kerr et al., Z. Phys. B 59, 103 (1985).

[16] T. S. Parker and L. O. Chua, Proc. IEEE 75, 982 (1987). 